ROCZNIKI HUMANISTYCZNE

Tom LXVIII, zeszyt 5 - 2020

DOI: http://dx.doi.org/10.18290/rh20685-16

TOMASZ NIESTOROWICZ

\title{
ACERCA DE LAS PROPIEDADES TEMPORALES Y ASPECTUALES DEL PERFECTO EN ESPAÑOL Y EN INGLÉS: UNA PERSPECTIVA DIDÁCTICA
}

\begin{abstract}
R e s u m e n. El propósito de este trabajo es describir algunas características temporales y aspectuales del perfecto en español y en inglés desde una perspectiva didáctica. Así pues presentaremos un estudio contrastivo en el cual intentamos analizar las formas verbales del pretérito perfecto compuesto en comparación con las formas verbales del present perfect inglés. La fundamentación de nuestro estudio viene motivada por la necesidad de reflexionar sobre la aplicación didáctica de la comparación en el caso de los aprendices de ELE cuya L1 es el polaco y los que tienen como referencia el inglés como L2, dado que, en polaco, al pretérito perfecto compuesto y al pretérito perfecto simple les corresponde la misma forma gramatical de pasado, así que el polaco carece de una oposición temporal semejante a la de pretérito perfecto compuesto y pretérito perfecto simple.
\end{abstract}

Palabras clave: tiempo; aspecto; perfecto/perfect; enseñanza de español como lengua extranjera (ELE).

\section{INTRODUCCIÓN}

En español, casi todas las formas compuestas pertenecen al ámbito temporal de las formas verbales relativas que indican una relación de anterioridad respecto del punto determinado establecido en el momento del habla (Cartagena 2997). En el caso del pretérito perfecto compuesto, se establecen las relaciones temporales de la anterioridad primaria respecto de una referencia simultánea al origen, definidas por la fórmula (OoV)-V (Rojo; Rojo \& Veiga). En las fórmulas de Bello (Análisis ideológica; Gramática de la len-

Dr Tomasz Niestorowicz - Universidad Católica de Lublin Juan Pablo II, Facultad de Humanidades, Departamento de Lingüística, Cátedra de Lingüística Románica; dirección para correspondencia: Aleje Racławickie 14, 20-950 Lublin; correo electrónico: tomasz.niestorowicz@kul.pl. ORCID: https://orcid.org/0000-0002-2849-5418. 
gua castellana), la combinación binaria de las relaciones temporales del pretérito perfecto compuesto se expresa mediante el término de ante-presente. En términos reichenbachianos (Alarcos Llorach), el pretérito perfecto compuesto, tanto en español como en inglés, se define por los símbolos E-H,R, ello significa, que el evento denotado por el predicado se sitúa en un punto anterior a un punto de referencia que es simultáneo al momento del habla.

Squartini y Bertinetto describen el fenómeno de la "deriva aorística" (aoristic drift), en el cual el pretérito perfecto compuesto va perdiendo su valor aspectual de perfecto para adquirir el valor aspectual de perfectividad (o aoristo) del pretérito perfecto simple (Azpiazu, "Del perfecto" 17). En la opinión de Azpiazu ("Del perfecto" 17), el valor perfectivo se refiere solo a la terminación del evento en el momento del habla, mientras que el valor aorístico, además de la terminación del evento, exige que un momento temporal esté desvinculado del presente. En otras palabras, como apunta la autora (Azpiazu, "Del perfecto" 18-19), los eventos vinculados temporalmente al presente ("presente extendido" de la NGLE o "presente ampliado" de Alarcos Llorach) serían los que se reflejan en la fórmula $(\mathrm{OoV})-\mathrm{V}$, mientras que los cuales no están vinculados al presente se refieren a la fórmula del pretérito perfecto simple: O-V (con valor aorístico) (cfr. Rojo; Rojo \& Veiga).

En los términos de sus valores aspectuales y temporales, el pretérito perfecto compuesto puede establecer una oposición con el pretérito perfecto simple, pero también con el presente ( $c f r$. Wilk-Racięska). En el estudio de Howe \& Rodríguez Louro, se observa la importancia de la semántica léxica del verbo (características modoaccionales), dentro de la distinción entre el pretérito perfecto compuesto y el presente. Por ejemplo, en el contexto, en el cual se emplea la frase adverbial desde hace X tiempo, el pretérito perfecto compuesto suele aparecer con los predicados télicos, mientras que en el caso de los predicados atélicos, la tendencia es doble: se ve la preferencia por el presente en los verbos de estado, en cambio, en cuanto a los verbos de actividad, no se observa una preferencia fuerte por una u otra forma (Howe 69).

En lo que se refiere a la noción de la proximidad temporal, Alarcos Llorach (33, citado por Veiga 166) sostiene que este fenómeno no debe ser considerado de manera absoluta en la distinción entre el pretérito perfecto compuesto y el pretérito perfecto simple. El autor destaca que el factor más importante es si el intervalo de tiempo en que ocurre el evento abarca o no el presente gramatical (Alarcos Llorach 33). Rojo expone que "hay que tener en cuenta, sin embargo, que esto no implica que lo indicado por llegué sea objetivamente anterior a lo expresado mediante he llegado. El hablante pue- 
de considerar lo simultáneo al origen tan amplio como desee: un día, un siglo o la historia humana" (105). En el ejemplo Toda la vida lo he creído un inútil, pero ayer me demostró su gran capacidad, el autor subraya que el evento expresado por demostró parece ser más próximo al origen que la forma he creido (Rojo 105).

La vinculación al momento presente es un rasgo definitorio del pretérito perfecto compuesto que consiste, entre otros, en la manifestación de las consecuencias de eventos pasados en el presente; además, la relevancia actual puede caracterizarse por un matiz subjetivo. Según Azpiazu, "por defecto todos los eventos sin CA se interpretan como vinculados al presente, y solo no lo están aquellos en los que la desvinculación se manifiesta expresamente por medio del adverbio" ("Del perfecto" 20), ya que los complementos adverbiales implican "presentar los hechos como factuales y susceptibles de constatación" ("Del perfecto" 23). El pretérito perfecto compuesto mantiene su relación temporal de simultaneidad, pero más bien en su aspecto pragmático o discursivo que el temporal; la simultaneidad objetiva se convierte en la "simultaneidad fingida" ("Del perfecto" 23-27). La forma compuesta introduce en el discurso los elementos más relevantes y novedosos, dinamiza y acerca la narración al momento del habla. Como apunta Wilk-Racięska (29), la vinculación al momento del habla se expresa mediante la proposición inferida de la proposición enunciada por medio del pretérito perfecto compuesto.

La categoría del perfecto no nos informa sobre la estructura interna de una situación, más bien muestra una relación de un estado con una situación anterior (Comrie 52). Rojo sitúa el perfecto "a caballo entre lo aspectual y lo temporal" (36). En lo concerniente a su valor temporal, el perfecto indica la relevancia actual de un evento anterior (Comrie 52). Klein (531) sostiene que no se puede definir la noción de la relevancia actual con criterios objetivos y la cuestión de determinar de manera precisa este fenómeno provoca muchas dificultades. El perfecto puede manifestarse de varias maneras: Comrie (56) propone cuatro tipos del perfecto, como expresión de los valores de resultado (perfect of result), continuidad (perfect of persistant situation), experiencia (perfect of experience) y pasado reciente (perfect of recent past); en nuestro análisis se seguirá la clasificación en cuestión. A continuación, presentaremos una serie de pautas y ejemplos que se centran en el contraste entre el pretérito perfecto compuesto español y el present perfect inglés y, por fin, reflexionaremos sobre la aplicación didáctica de tal estudio comparativo. 


\section{LAS LECTURAS DEL PERFECTO \\ EN ESPAÑOL Y EN INGLÉS}

Comrie define el perfecto de resultado como la persistencia de un resultado producido por un evento pasado en el momento del habla, como en el ejemplo (56):

(1) ¿Está Juan aquí ya? - Sí, ha llegado.

* - Sí, llegó.

Is John here yet? - Yes, he has arrived.

* - Yes, he arrived.

En el ejemplo (1) se ilustra, por medio del pretérito perfecto compuesto, que el sujeto "sigue estando aquí" en el momento del habla. La lectura resultativa aparece frecuentemente con predicados télicos (lo indicado por ha llegado). En este caso, el uso de la forma verbal en cuestión en español coincide con el uso en inglés.

En el caso del perfecto de continuidad, un evento que empezó antes del momento del habla puede continuar hasta el momento de la enunciación, como en We've lived here for ten years (Comrie 60). En el ejemplo mencionado en Brucart \& Xiqués (11), en español se prefiere el tiempo presente en vez del pretérito perfecto compuesto:

(2) Vivo en Londres desde 2015.

I have lived in London since 2015.

*I live in London since 2015.

Como se observa en el ejemplo (2), en inglés la lectura continuativa no es posible en el presente. En español, la lectura universal del pretérito perfecto compuesto, en este contexto, en el cual se remite a un momento determinado en el pasado, es muy limitada y se prefiere emplear las formas verbales del presente. En cambio, en los siguientes ejemplos se indica más bien la duración y el complemento adverbial no remite a un momento específico:

(3) Hemos vivido (durante) diez años en Londres.

We have lived in London for ten years.

(4) Siempre han vivido en Barcelona.

They have always lived in Barcelona. 
Obsérvese que al perfecto de continuidad se le puede atribuir un valor aspectual imperfectivo, además, se realiza este por medio de predicados atélicos (lo expresado mediante hemos vivido y han vivido en los ejemplos (3) y (4), respectivamente), los cuales se denotan como durativos o repetitivos (Howe 65). La doble orientación temporal se refleja en la descripción de los valores aspectuales del pretérito perfecto compuesto como los imperfectivos y perfectivos a la vez. En la oración He trabajado veinte años para él se puede tener en cuenta dos interpretaciones: "sigo trabajando para él" muestra el valor imperfectivo del "antepresente continuo", mientras que "ya no trabajo para él" representa el valor perfectivo del "antepresente no continuo" (NGLE 1726; Veiga 156).

El perfecto de experiencia indica un evento que ha ocurrido antes del momento del habla en el intervalo del tiempo que se extiende hasta el momento de la enunciación (Bill has been to America) (Comrie 59). Según Comrie, esto indica que una situación ocurrió por lo menos una vez en el pasado, como en:

(5) He estado en Salamanca.

I have been to Salamanca.

Como apunta Dahl (citado por Soto 133), este tipo del perfecto puede caracterizarse por la repetibilidad en el momento de la enunciación, así que puede reflejar un evento genérico que se centra en un tipo (type) de evento (he estado), mientras que el uso del pretérito perfecto simple remitiría a un caso (token) de un evento (estuve) (Dahl y Hedin). En las oraciones del tipo:

(6) Mi abuela ha muerto.

My grandmother has died.

se trata, como indica la NGLE (1725), de "cierto bagaje de conocimientos o de vivencias que caracterizan a los individuos". El ejemplo "puede verse como el resultado natural de insertar el suceso de morir alguien en el período implícito representado por la vida de esa otra persona hasta el momento del habla". En los ejemplos (5) y (6) coinciden el español y el inglés.

El perfecto de pasado reciente remite a un evento que, por la proximidad temporal al momento del habla, posee el rasgo de la relevancia actual (I have recently learned that the match is to be postponed). La relevancia actual de un evento no necesariamente implica que este se haya producido recientemente (Comrie 60). En la opinión de Gutiérrez Araus, la distancia cronológi- 
ca no es un criterio decisivo a la hora de elegir las formas del pretérito perfecto compuesto o del pretérito perfecto simple, sino la orientación de un intervalo de tiempo en relación a una perspectiva temporal actual.

Comrie (54-61) aduce ejemplos de la lectura hodiernal, en los cuales en español se emplea el pretérito perfecto compuesto para referirse a un evento que ha ocurrido antes del momento del habla y el momento del habla es la tarde o la noche del mismo día:

(7) Me he levantado a las cinco. ?Me levanté a las cinco.

*I have got up at five o'clock.

(8) La he visto esta mañana.

?La vi esta mañana.

*I have seen her this morning.

Observemos que en los ejemplos (7) y (8) se utilizarían en inglés las formas verbales de past simple, dado que el present perfect admite la especificación temporal solo en el ejemplo I have (on some occasion in the past) got up at five o'clock pero se trata de una lectura iterativa y de todos los posibles five o'clocks. También es aceptable $I$ have seen her this morning a condición de que el enunciado se produzca por la mañana (Comrie 54-61). Klein (544-545) sugiere que en inglés existe la constricción position-definiteness que se refleja en el fenómeno de Present Perfect Puzzle. Según el autor, la noción de position-definiteness constraint denota, de manera estricta, la localización de un evento en el eje temporal. Por lo tanto, el Tiempo del Tópico y el Tiempo de la Situación no pueden ser independientemente especificados en cuanto a su localización temporal. En el ejemplo (7), el Tiempo de la Situación está limitado por el complemento adverbial at five, así que posee el estatus de positiondefinite, mientras que el Tiempo del Tópico es especificado por el morfema del presente have. En español, la constricción position- definiteness no existe, por eso el complemento adverbial esta mañana puede combinarse libremente con el morfema del presente he. Huelga decir que, en inglés, el llamado intervalo temporal de perfecto tiene las mismas propiedades funcionales que el presente: es un intervalo temporal sin límites claramente determinados (a la izquierda y a la derecha) y, por eso, no puede coincidir con un momento especificado. En español, el intervalo es más extendido y relacionado con la situación presente, pero puede incluir momentos localizados en el pasado que pertenecen al momento presente (Azpiazu, "Simultaneity" 125). Las oraciones del 
tipo Me levanté a las cinco para referirse a un evento que ha ocurrido antes del momento del habla y el momento del habla es la tarde o la noche del mismo día, sería aceptable en las variedades extrapeninsulares. En el ejemplo de Kempas (52), el predicado designa un evento que ha ocurrido en el pasado reciente:

(9) No he oído lo que has dicho.

*I haven't heard what you have said.

En este caso, en la variedad peninsular estándar se emplea el pretérito perfecto compuesto, mientras que las construcciones del tipo No oi lo que dijiste puede ser admitida solo en la zona del noroeste peninsular. En inglés, la oración *I haven't heard what you have said sería agramatical, en este contexto solo sería aceptable el uso de past simple, como en I didn't hear what you said. El present perfect inglés tampoco es compatible con complementos adverbiales (los ejemplos: (10) y (11) de Klein 525) que localizan un evento en el pasado (yesterday, two hours ago) por la existencia del llamado position-definiteness:

(10) *Ayer Chris ha salido de York.

*Chris has left York yesterday.

(11) Chris ha salido hace dos horas. *Chris has left two hours ago.

Cabe subrayar que en español el uso prehodiernal del pretérito perfecto compuesto aorístico en el ejemplo (10) (*Ayer Chris ha salido de York) no es aceptable en la variedad peninsular (pero sí en algunas variedades extrapeninsulares, $c f r$. Kempas), aunque en algunos casos se puede justificar el uso del pretérito perfecto compuesto en un contexto determinado, en la opinión de Azpiazu y Kempas (723): "defendemos que la gradación en la aceptabilidad no tiene tanto que ver con el hecho de que el evento esté más o menos alejado en el tiempo respecto al origen (o momento elocutivo), ni con la mera presencia de CCAA localizadores en puntos concretos del pasado, sino con la configuración misma (semántica y sintáctica) del discurso en el que se inserta la forma: posición del CA, tipo de CA, tipo de evento". La mayoría de los ejemplos del uso prehodiernal, investigados por los autores, cumple las condiciones siguientes: tenemos que ver con los predicados télicos con lectura resultativa, además, los complementos adverbiales pospuestos indican la localización temporal más bien inconcreta con hace ( hace poco, hace años) (Azpiazu y Kempas 722). 


\section{CONCLUSIONES}

A nuestro modo de ver, lo importante es reconocer que solo algunos de los valores temporales y aspectuales del pretérito perfecto compuesto español y del present perfect inglés son convergentes. En lo concerniente a la lectura resultativa, el empleo de ambas formas verbales coincide: tienden a expresar lo resultativo con los predicados télicos. Para expresar situaciones aún no concluidas en el presente y que, teóricamente, pueden transcender al futuro, en español, de manera natural, se utilizan las formas verbales del presente, a no ser que en el contexto aparezcan complementos adverbiales no específicos y/o durativos. En cambio, en inglés, para formular la lectura continuativa no es posible la aparición del tiempo presente y se exige el empleo del present perfect. Lo que tienen en común el pretérito perfecto compuesto español y el present perfect inglés en el caso del perfecto de experiencia son los siguientes rasgos: la noción de repeatability, es decir, la inferencia de que un evento es genérico además, un matiz subjetivo de la relevancia actual. Se ven diferencias en la lectura hodiernal por la existencia del fenómeno de position-definiteness que restringe la aparición del present perfect con ciertos complementos adverbiales localizadores en la línea temporal del pasado, lo que no ocurre en el caso del pretérito perfecto compuesto. Por fin, hay puntos de convergencia en cuanto al uso prehodiernal, que está limitado en español a unos contextos determinados y algunas variedades diatópicas.

Puesto que en el sistema verbal polaco al pretérito perfecto compuesto y al pretérito perfecto simple les corresponde la misma forma gramatical de para un aprendiente polaco es difícil diferenciar las formas verbales en cuestión. En los manuales de ELE se enumeran principalmente los complementos adverbiales característicos del pretérito perfecto compuesto y se comparan con los típicos del pretérito perfecto simple.

La reflexión metalingüística y el desarrollo del conocimiento explícito desempeñan el papel fundamental en el proceso de adquisición /aprendizaje de una segunda lengua. A veces los aprendices de una segunda lengua entienden mejor el sistema gramatical que sus compañeros nativos, entonces, su conocimiento explícito es muy superior pero esto no ocurre en el caso del conocimiento instrumental. Una persona que aprende un segunda lengua tiene como el punto de referencia su lengua materna, en la que principalmente construye su conciencia metalingüística. Pero en las etapas posteriores del aprendizaje, una segunda lengua como el inglés puede contribuir a crear la posibilidad de analizar las estructuras morfosintácticas mediante el análisis 
contrastivo, particularmente nos referimos a los estudiantes de nivel universitario. Es muy importante analizar las formas gramaticales mediante ejercicios contextualizados; gracias a ello se puede observar las relaciones entre la forma, el significado y el uso adecuado en un contexto determinado.

\section{TRABAJOS CITADOS}

Alarcos Llorach, Emilio. "Perfecto simple y compuesto en español." Revista de Filología Española, vol. 31, 1947, pp. 108-139.

Azpiazu, Susana. "Del perfecto al aoristo en el antepresente peninsular: un fenómeno discursivo." Formas simples y compuestas de pasado en el verbo español, edited by Susana Azpiazu, Axac, 2014, pp. 17-30.

Azpiazu, Susana "Simultaneity and 'increased present' in the European Spanish perfect." Catalan Journal of Linguistics, vol. 17, 2018, pp. 117-134.

Azpiazu, Susana, and Ilpo Kempas. "Acerca del uso prehodiernal del pretérito perfecto compuesto resultativo en español." Zeitschrift für romanische Philologie, vol. 133, no. 3, 2017, pp. 709-727.

Bello, Andrés. Análisis ideológica de los tiempos de la conjugación castellana. Imprenta de M. Rivadeneyra, 1841.

Bello, Andrés. Gramática de la lengua castellana destinada al uso de los americanos. Imprenta del Progreso, 1847.

Brucart, Josep, and Teresa M. Xiqués, T. "Some issues about the perfect." Catalan Journal of Linguistics, vol. 17, 2018, pp. 5-18.

Cartagena, Nelson. "Los tiempos compuestos". Gramática descriptiva de la lengua española, edited by Ignacio Bosque and Violeta Demonte, Espasa-Calpe, 1999, pp. 2933-2975.

Comrie, Bernard. Aspect. Cambridge UP, 1976.

Dahl, Osten. Tense and aspect systems. Basil Blackwell,1985.

Dahl, Osten, and Eva Hedin. "Current Relevance and Event Reference." Tense and Aspect in the Languages of Europe, edited by Osten Dahl, De Gruyter, 2000, pp. 385-402.

Gutiérrez Araus, María Luz. Formas temporales del pasado en indicativo. Arco Libros, 1995.

Howe, Chad. "Variación y divergencia en el desarrollo del pasado perifrástico en las lenguas románicas." Formas simples y compuestas de pasado en el verbo español, edited by Susan Azpiazu, Axac, 2014, pp. 63-79.

Howe, Chad, and Celeste Rodríguez Louro. "Peripheral envelopes: Spanish perfects in the variable context." Selected Proceedings of the 6th Workshop on Spanish Sociolinguistics, edited by Ana M. Carvalho and Sara Beaudrie, Cascadilla Proceedings Project, 2013, pp. 41-52.

Kempas, Ilpo. Estudio sobre el uso prehodiernal del Pretérito Perfecto en el español peninsular y en comparación con la variedad del español argentino hablada en Santiago del Estero, tesis doctoral, Universidad de Helsinki, 2006.

Klein, Wolfgang. "The present perfect puzle". Language, vol. 68, no. 3, 1992, pp. 525-552.

$N G L E=$ RAE \& AALE. Nueva gramática de la lengua española. Espasa-Calpe, 2009.

Nowikow, Wiaczesław. "Tiempos verbales". Gramática contrastiva español-polaco, edited by Wiaczesław Nowikow, Wydawnictwo Uniwersytetu Łódzkiego, 2017, pp. 127-178.

Rojo, Guillermo. “La temporalidad verbal en español.” Verba, vol. 1, 1974, pp. 68-149. 
Rojo, Guillermo, and Alexandre Veiga. "El tiempo verbal. Los tiempos simples." Gramática descriptiva de la lengua española, edited by Ignacio Bosque and Violeta Demonte, EspasaCalpe, 1999, pp. 2866-2933.

Soto, Guille. "El pretérito perfecto compuesto en el español estándar de nueve capitales americanas: frecuencia, subjetivización y deriva aorística." Formas simples y compuestas de pasado en el verbo español, edited by Susana Azpiazu, Axac, 2014, pp. 131-146.

Squartini, Mario, and Pier Marco Bertinetto. "The Simple and Compound Past in Romance Languages." Tense and Aspect in the Languages of Europe, edited by Osten Dahl, De Gruyter, 2000, pp. 403-439.

Veiga, Alexandre. "La anterioridad del «pretérito perfecto»." Formas simples y compuestas de pasado en el verbo español, edited by Susana Azpiazu, Axac, 2014, pp. 147-177.

Wilk-Racięska, Joanna. El tiempo interior: una aproximación al aspecto en español. Wydawnictwo Uniwersytetu Śląskiego, 2004.

\section{WARTOŚCI TEMPORALNE I ASPEKTUALNE \\ KATEGORII PERFECTO / PERFECT W JĘZYKU HISZPAŃSKIM I ANGIELSKIM: PERSPEKTYWA DYDAKTYCZNA}

$$
\text { Streszczenie }
$$

Celem niniejszego artykułu jest opis cech temporalnych i aspektualnych kategorii perfecto / perfect $\mathrm{w}$ języku hiszpańskim i angielskim $\mathrm{z}$ perspektywy dydaktycznej. Zaprezentowane zostanie kontrastywne studium, w którym porówna się formy werbalne hiszpańskiego czasu pretérito perfecto compuesto $\mathrm{z}$ formami werbalnymi angielskiego czasu present perfect. Artykuł motywowany jest potrzebą refleksji nad dydaktycznym zastosowaniem niniejszego studium komparatywnego dla polskich studentów uczących się języka hiszpańskiego, dla których jedynym punktem odniesienia może być język angielski, zważywszy, że w języku polskim nie istnieje opozycja czasowa typu pretérito perfecto compuesto / pretérito perfecto simple.

Słowa kluczowe: czas; aspekt; perfecto / perfect; nauczanie języka hiszpańskiego jako języka obcego (ELE).

\section{TEMPORAL AND ASPECTUAL PROPERTIES OF THE CATEGORY PERFECTO / PERFECT IN SPANISH AND ENGLISH: A DIDACTIC PERSPECTIVE}

\section{S u m mary}

The purpose of the present article is to describe the temporal and aspectual characteristics of the category perfecto / perfect in Spanish and English from a didactic perspective. A contrastive study will be presented, which will compare the verbal forms of Spanish pretérito perfecto compuesto tense with verbal forms of English present perfect tense. The present article is motivated by the need to reflect on the didactic use of the comparative study for Polish students learning Spanish as a foreign language, for whom the only reference point may be English, given that there is no temporal opposition in Polish such as pretérito perfecto compuesto / pretérito perfecto simple.

Key words: tense; aspect; perfecto / perfect; teaching Spanish as a foreign language (ELE). 